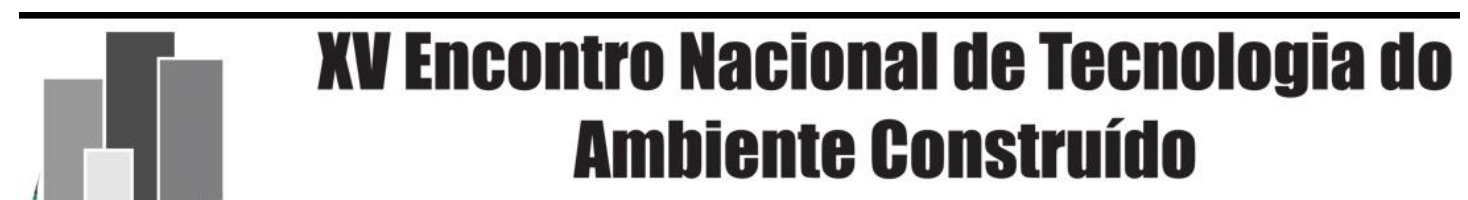

Avanços no desempenho das construções - pesquisa, inovação e capacitação profissional

ENTAC 12,13 E 14 DE NOVEMBRO DE 2014 | MACEIÓ | AL

\title{
UTILIZAÇÃO DE RESÍDUO DE SERRAGEM DE ROCHAS ORNAMENTAIS COMO AGREGADO MIÚDO, EM CONCRETO
}

\author{
ROQUE, Alex Borges (1); CAMPOS, Rafael Nascimento (2); SANTOS, Heni \\ Mirna Cruz (3); MOURA, Washington Almeida (4)
}

(1) Universidade Estadual de Feira de Santana, e-mail: alex.borgez@gmail.com (2) Universidade Estadual de Feira de Santana, e-mail: rafaelcamposeng@gmail.com (3) Universidade Estadual de Feira de Santana, e-mail: henimirna@hotmail.com (4) Universidade Estadual de Feira de

Santana, e-mail: washington.moura@gmail.com

\begin{abstract}
RESUMO
A crescente preocupação com as questões ambientais envolvendo consumo de recursos naturais e destinação dos resíduos sólidos industriais tem levado a realização de várias pesquisas com vistas a redução do consumo de matérias-primas, bem como uma adequada destinação destes resíduos. No processo de produção de rochas ornamentais, por exemplo, é gerada uma grande quantidade de resíduos. O Brasil, que é um dos grandes produtores de rochas ornamentais, tem convivido com este problema. Uma das possibilidades de aproveitamento de resíduos estudadas visa a sua incorporação na construção civil, por apresentar muitas opções para o reaproveitamento, devido à variedade de produtos que a mesma consome. O presente trabalho verificou a influência do resíduo de serragem de rochas ornamentais (RSRO) nas propriedades do concreto. O RSRO foi utilizado em substituição parcial do agregado miúdo no concreto, nos teores de $10 \%$ e $20 \%$, em massa. A avaliação foi feita com base nos resultados de resistência à compressão axial, resistência à tração por compressão diametral e absorção por imersão. Observou-se que a utilização do resíduo proporcionou um aumento nas resistências à compressão axial e tração e redução da absorção dos concretos, em relação ao de referência. Com base nos resultados podese recomendar a utilização de até $20 \%$ do RSRO, em substituição ao agregado miúdo natural.
\end{abstract}

Palavras-chave: Resíduo de serragem de rochas ornamentais, reciclagem, resistências mecânicas, absorção.

\begin{abstract}
The environmental issues linked to natural resource consumption and proper disposal of industrial solid waste represent driving forces to several research programs. The dimension stone production process, for example, generates a large amount of waste. Brazil, which is one of the major producers of dimension stone, has faced problems with the dimension stone sawing dust. Incorporating this type of waste into the construction industry represents a good alternative to minimize the problem. So, the present study examined the influence of the dimension stone sawing dust (DSSD) on the concrete properties. The DSSD was used in partial replacement of the natural fine aggregate (0 and 20\%, by mass) to concrete production. The evaluation was made based on the results of compressive strength, splitting tensile strength, and water absorption rate. It was observed that the use of the DSSD promoted an increase of the compressive and splitting tensile strength of DSSD concrete. Also, a water absorption rate reduction was observed when compared to the reference concrete. Based on the results, it is possible to stand the use of up to $20 \%$ of DSSD, in replacement of natural fine aggregate.
\end{abstract}

Keywords: Sawing waste of dimension stone, recycling, mechanical resistance, absorption. 


\section{INTRODUÇÃO}

O setor da construção civil é uma das grandes consumidoras de recursos naturais e também uma grande geradora de resíduos sólidos. Neste contexto, um dos desafios enfrentados atualmente por este setor industrial é a relação entre o desenvolvimento tecnológico e a preservação ambiental. Este setor apresenta uma grande versatilidade de produtos e de processos produtivos, sendo então uma alternativa para o aproveitamento de resíduos sólidos como matéria-prima suplementar na sua produção.

A indústria de rochas ornamentais tem uma significativa participação na economia brasileira, sendo responsável pelo faturamento de US\$ 1,1 bilhão no ano de 2012 (CHIODI FILHO, 2013). As rochas ornamentais e de revestimento abrangem as rochas que podem ser extraídas em blocos ou placas, cortadas de diversas formas e que passam por um beneficiamento de suas faces através de processos de esquadrejamento, polimento, lustro, entre outros (CHIODI FILHO, 2001). Nesse processo de beneficiamento das rochas ornamentais, há geração de um grande volume de resíduos. Este resíduo pode ser proveniente da extração do bloco, da serragem para padronização, do processo de corte e polimento além dos finos da lavra e do beneficiamento (MOURA \& LEITE, 2011). No Brasil, esses resíduos têm tido, na maioria das vezes, uma deposição inadequada no meio ambiente, resultando em impactos que podem comprometer flora e fauna (MANHÃES \& HOLANDA, 2008). A serragem dos blocos pode ser feita utilizando polpa abrasiva (para minimizar o desgaste da lâmina) ou fio diamantado. A polpa abrasiva é composta por água, granalha (aço), cal e rocha moída e, geralmente, é lançada, por bombeamento, sobre o bloco, através chuveiros (SILVA, 1998).

O RSRO tem sido objeto de diversos estudos (MOURA, GONÇALVES \& LEITE, 2002; MOURA et al., 2009; BARROS, 2008) que apontam para um potencial de utilização desse resíduo como substituição parcial do cimento e do agregado miúdo em concretos e argamassas.

O presente trabalho visa avaliar a influência nas características de concretos contendo o resíduo de serragem de rochas ornamentais (RSRO), obtidas pelo processo de corte através de polpa abrasiva (PA), como substituto parcial do agregado miúdo. Para isso, foram produzidos concretos contendo $0 \%$ (referência) $10 \%$ e $20 \%$ de RSRO-PA, substituindo o agregado miúdo, em massa e verificadas as propriedades: trabalhabilidade, resistência à compressão axial, resistência à tração por compressão diametral e absorção de água por imersão.

\section{MATERIAIS E MÉTODOS}

\subsection{Caracterização dos materiais}

Para a produção dos concretos, foram utilizados o cimento CP II Z 32 - RS (por ser um dos mais utilizados na região de Feira de Santana), resíduo de serragem de rochas ornamentais obtido por corte utilizando polpa abrasiva (RSRO-PA), areia fina proveniente do município de Alagoinhas-BA e brita granítica proveniente da pedreira Rio Branco, situada em Feira de Santana, na Bahia.

\subsubsection{Cimento Portland}

Os resultados dos ensaios de caracterização são apresentados a seguir na Tabela 1. 
Tabela 1 - Características do cimento

\begin{tabular}{c|c|c}
\hline \multicolumn{2}{c|}{ Características e Propriedades } & $\begin{array}{c}\text { Valores } \\
\text { experimentais }\end{array}$ \\
\hline \multirow{2}{*}{$\begin{array}{c}\text { Resistência à compressão axial } \\
(\mathrm{MPa})\end{array}$} & 3 dias de idade & 26,5 \\
\cline { 2 - 3 } & 7 dias de idade & 29,1 \\
\cline { 2 - 3 } & 28 dias de idade & 34,9 \\
\hline Massa Específica $\left(\mathrm{g} / \mathrm{cm}^{3}\right)$ & 2,90 \\
\hline \multicolumn{2}{c}{ Finura $(\%)$} & 0,5 \\
\hline
\end{tabular}

O cimento utilizado atende os requisitos da NBR 11578 (ABNT, 1991).

\subsubsection{Resíduo de serragem de rochas ornamentais - RSRO-PA}

Foi utilizado neste trabalho, o mesmo RSRO-PA utilizado no trabalho desenvolvido por Silva, Santos \& Moura (2013). Nas Tabelas 2 e 3 encontram-se os resultados dos ensaios de caracterização física e química do resíduo, respectivamente.

Tabela 2 - Caracterização física do RSRO-PA

\begin{tabular}{c|c|c|c}
\hline Resíduo & $\begin{array}{c}\text { Massa Específica } \\
\left(\mathbf{g} / \mathbf{c m}^{\mathbf{3}}\right)\end{array}$ & $\begin{array}{c}\text { Massa Unitária } \\
\left(\mathbf{k g} / \mathbf{d m}^{3}\right)\end{array}$ & $\begin{array}{c}\text { Diâmetro Médio } \\
(\boldsymbol{\mu m})\end{array}$ \\
\hline RSRO & 2,74 & 1,01 & 6,12 \\
\hline
\end{tabular}

Fonte: (SILVA, SANTOS \& MOURA, 2013).

Tabela 3 - Composição Química do RSRO-PA

\begin{tabular}{|c|c|c|c|c|c|c|c|c|c|}
\hline $\begin{array}{c}\mathrm{SiO}_{2} \\
(\%)\end{array}$ & $\begin{array}{c}\mathbf{A l}_{2} \mathbf{O}_{3} \\
(\%)\end{array}$ & $\begin{array}{c}\operatorname{MgO} \\
(\%)\end{array}$ & $\begin{array}{l}\mathbf{K}_{2} \mathbf{O} \\
(\%)\end{array}$ & $\begin{array}{c}\mathrm{TiO}_{2} \\
(\%)\end{array}$ & $\begin{array}{c}\mathrm{Na}_{2} \mathrm{O} \\
(\%)\end{array}$ & $\begin{array}{c}\mathrm{Fe}_{2} \mathbf{O}_{3} \\
(\%)\end{array}$ & $\begin{array}{l}\mathrm{SO}_{4} \\
(\%)\end{array}$ & $\begin{array}{c}\mathrm{CaO} \\
(\%)\end{array}$ & $\begin{array}{c}\text { Perda ao } \\
\text { Fogo } \\
(\%)\end{array}$ \\
\hline 5,893 & 1,231 & 0,397 & - & 0,350 & 0,298 & 9,060 & 0,400 & 79,010 & 0,211 \\
\hline
\end{tabular}

Fonte: (SILVA, SANTOS \& MOURA, 2013).

\subsubsection{Agregado miúdo}

O agregado miúdo utilizado trata-se de uma areia fina, proveniente do município de Alagoinhas-BA, cuja dimensão máxima característica foi de 1,2mm e massa específica, determinada segundo a NM 52 (ABNT, 2009), igual a 2,64g/ $\mathrm{cm}^{3}$.

\subsubsection{Agregado graúdo}

Foi utilizado como agregado graúdo uma brita de origem granítica, oriunda da pedreira Rio Branco, em Feira de Santana, Bahia. A massa específica, determinada através da NM 53 (ABNT, 2003), foi de 2,73 $\mathrm{g} / \mathrm{cm}^{3}$ e a massa unitária, determinada através da NBR 7251 (ABNT, 1982), foi igual a $1,47 \mathrm{~kg} / \mathrm{dm}^{3}$. 


\subsection{Produção dos concretos}

Os concretos foram produzidos no LaboTec (Laboratório de Tecnologia) da UEFS utilizando uma betoneira elétrica de $220 \mathrm{~V}$, capacidade de $145 \mathrm{~L}$ e rotação de $34 \mathrm{RPM}$. Foi fixado a relação a/c em 0,54 . Na Tabela 4 estão apresentados os traços produzidos.

Tabela 4 - Traços dos concreto produzidos

\begin{tabular}{c|c}
\hline Concreto & Traço \\
\hline Referência & $1: 1,94: 3,06: 0,54$ (cimento:areia:brita:água) \\
\hline 10\% RSRO-PA & $1: 0,19: 1,75: 3,06: 0,54$ (cimento:RSRO:areia:brita:água) \\
\hline 20\% RSRO-PA & $1: 0,38: 1,55: 3,06: 0,54$ (cimento:RSRO:areia:brita:água) \\
\hline
\end{tabular}

\section{APRESENTAÇÃO E DISCUSSÕES DOS RESULTADOS}

\subsection{Trabalhabilidade}

A trabalhabilidade foi medida através do ensaio de abatimento de tronco de cone, realizado de acordo com a NBR NM 67 (ABNT, 1998). Na Figura 1 são apresentados os resultados deste ensaio.

\section{Figura 1 - Resultado de abatimento do tronco de cone}

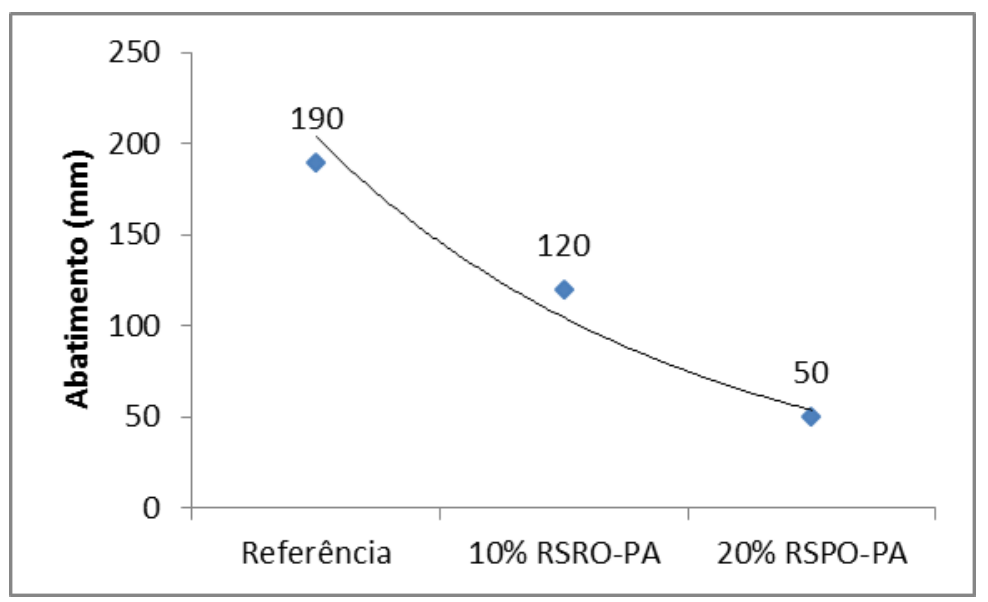

Foi verificada uma redução no abatimento dos concretos contendo RSRO-PA em relação ao concreto de referência. Essa redução foi de 36,84\% para a amostra contendo $10 \%$ de RSRO-PA e de $73,68 \%$ para a amostra contendo $20 \%$ do resíduo. A redução no abatimento pode ser explicada devido à finura do RSRO-PA que é muito maior do que a finura do agregado miúdo natural, necessitando, desta forma, de mais água para molhar a superfície dos grãos. Apesar da grande redução no abatimento, os concretos aparentavam boa trabalhabilidade e coesão. Logo, para que se obtenha o mesmo abatimento do concreto de referência faz-se necessário a utilização de aditivos superplastificantes.

\subsection{Resistência à compressão axial}

Os ensaios de resistência à compressão axial foram realizados de acordo com a norma NBR 5739 (ABNT, 2007). Foram ensaiados 4 corpos-de-prova de cada traço aos 28 dias. Os resultados de resistência à compressão estão apresentados na Tabela 5 . 
Tabela 5 - Resultados de resistência à compressão axial

\begin{tabular}{c|c|c|c}
\hline Amostra & $\begin{array}{c}\text { Resistência } \\
\text { (MPa) }\end{array}$ & $\begin{array}{c}\text { Desv. } \\
\text { Padrão }\end{array}$ & $\begin{array}{c}\text { C.V. } \\
(\mathbf{\%})\end{array}$ \\
\hline Referência & 23,80 & 0,61 & 2,56 \\
\hline 10\% RSRO-PA & 31,86 & 0,54 & 1,7 \\
\hline 20\% RSRO-PA & 32,65 & 1,85 & 5,68 \\
\hline
\end{tabular}

A Figura 2 apresenta o gráfico de comportamento dos concretos quanto à resistência à compressão axial.

Figura 2 - Comportamento dos concretos quanto à compressão axial

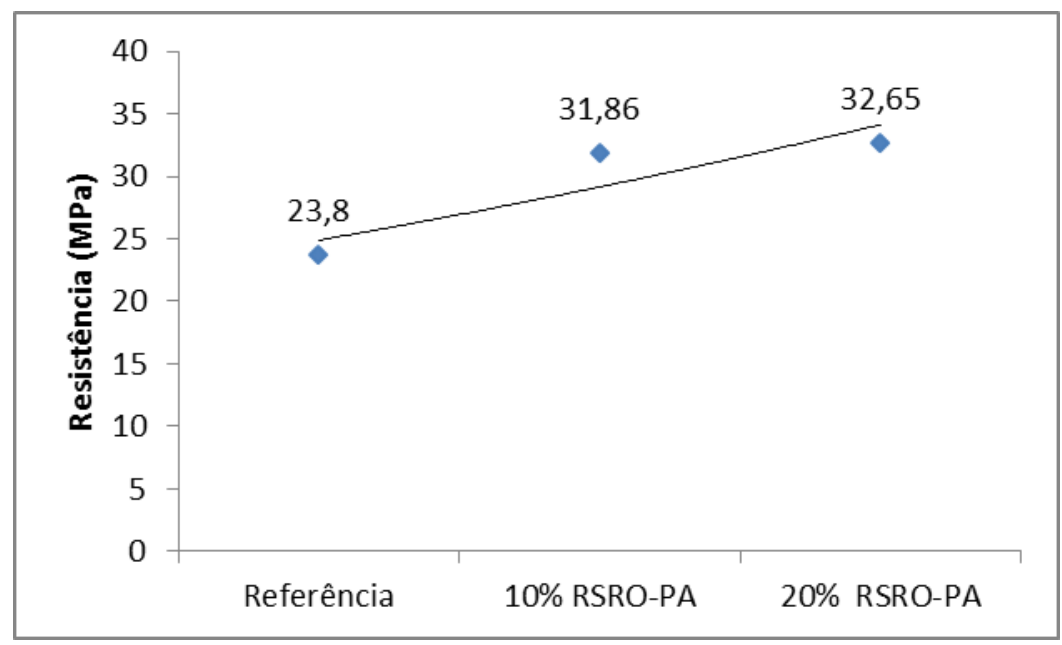

Pode-se observar que os concretos com RSRO-PA apresentaram melhor comportamento quanto à resistência à compressão axial, em relação ao concreto de referência.

Verificou-se um aumento de $33,87 \%$ e de $37,18 \%$ na resistência à compressão dos concretos contendo $10 \%$ e $20 \%$ de RSRO-PA, respectivamente, em relação ao concreto de referência. Este comportamento pode ser explicado devido o efeito de preenchimento. Como o diâmetro médio do RSRO-PA é de $6,12 \mu \mathrm{m}$, menor do que o próprio cimento, é possível que tenha ocorrido o efeito físico de melhor preenchimento de vazios. As partículas de RSRO podem ter contribuído também ter atuado como ponto de nucleação e favorecido à aceleração da formação de produtos hidratados a partir da matriz cimentícia.

No estudo desenvolvido por Moura, Gonçalves \& Leite (2002), que verificaram o comportamento de argamassas contendo $5 \%$ e $10 \%$ de RSRO em substituição ao agregado miúdo natural em argamassas, foi observado um incremento na resistência à compressão nas misturas contendo o RSRO. Os autores também atribuíram tal comportamento a maior finura do resíduo, proporcionando melhor efeito de preenchimento.

\subsection{Resistência à tração por compressão diametral}

Os ensaios de resistência à tração indireta foram realizados de acordo com a norma NBR 7222 (ABNT, 2011). Foram ensaiados 4 CP's cilíndricos de cada traço, aos 28 dias de idade. Na Tabela 6 estão apresentados os resultados de resistência à tração por compressão diametral. 
Tabela 6 - Resultados de resistência à tração por compressão diametral

\begin{tabular}{c|c|c|c} 
Amostra & $\begin{array}{c}\text { Resistência } \\
(\mathbf{M P a})\end{array}$ & $\begin{array}{c}\text { Desv. } \\
\text { Padrão }\end{array}$ & $\begin{array}{c}\text { C.V. } \\
(\boldsymbol{\%})\end{array}$ \\
\hline Referência & 2,06 & 0,05 & 2,26 \\
\hline 10\% RSRO-PA & 2,49 & 0,18 & 4,12 \\
\hline $20 \%$ RSRO-PA & 2,54 & 0,13 & 5,12 \\
\hline
\end{tabular}

A Figura 3 mostra o comportamento dos concretos quanto à resistência à tração por compressão diametral.

Figura 3 - Comportamento dos concretos em relação à tração por compressão diametral

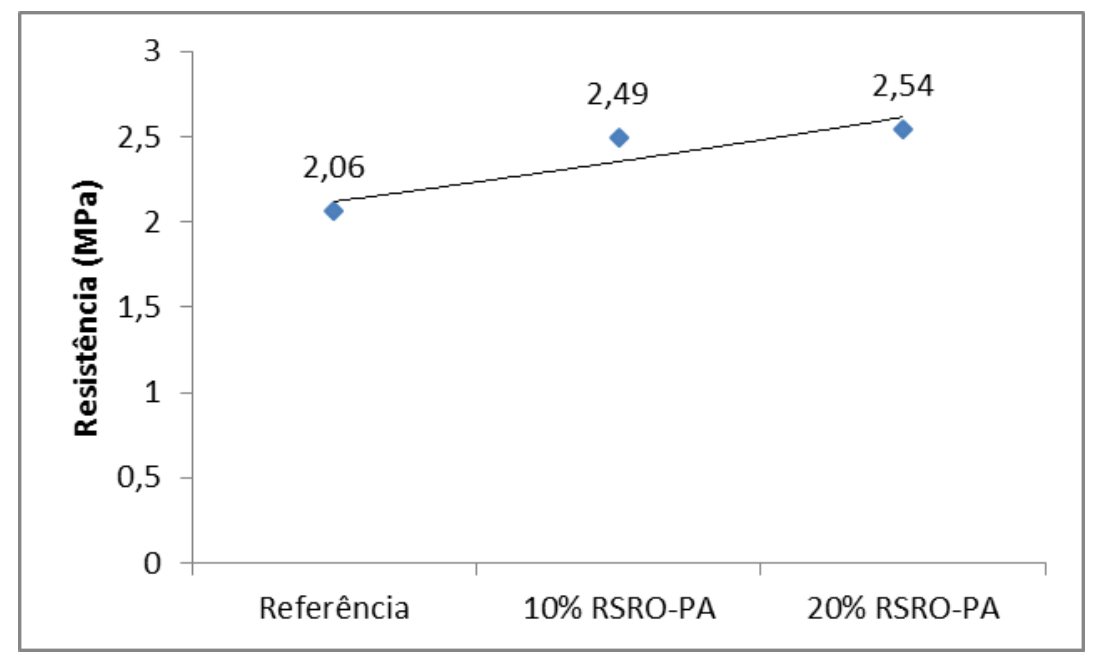

Observa-se que os concretos com RSRO-PA apresentaram maior resistência à tração por compressão diametral. O melhor resultado foi o do concreto com $20 \%$ do resíduo que proporcionou um aumento de $23,3 \%$, em relação ao concreto de referência.

O comportamento dos concretos quanto à resistência à tração foi semelhante ao de resistência à compressão axial.

De acordo com Mehta \& Monteiro (2008), à medida que a resistência à compressão aumenta, a resistência a tração também aumenta, porém a uma taxa decrescente, ou seja, quanto maior a resistência à compressão, menor a razão. Neville (1997), afirma que as resistências à tração e à compressão estão relacionadas, porém não existe uma proporcionalidade direta, a relação entre as duas resistências depende do nível de resistência do concreto. Quando a resistência à compressão aumenta, a resistência à tração também aumenta, mas a uma razão decrescente.

\subsection{Absorção}

O ensaio de absorção por imersão foi executado de acordo com a NBR 9778 (ABNT, 2005). Foram ensaiados 3 corpos de prova para cada traço de concreto, aos 28 dias. Os resultados do ensaio de absorção por imersão estão apresentados na Tabela 7. 
Tabela 7 - Resultados do ensaio de absorção por imersão

\begin{tabular}{c|c|c|c}
\hline Amostra & Absorção (\%) & Desv. Padrão & C.V. (\%) \\
\hline Referência & 5,42 & 0,24 & 4,42 \\
\hline 10\% RSRO-PA & 5,28 & 0,12 & 2,2 \\
\hline 20\% RSRO-PA & 5,06 & 0,18 & 3,47 \\
\hline
\end{tabular}

A Figura 4 mostra o comportamento dos concretos quanto à absorção por imersão

Figura 4 - Comportamento dos concretos quanto à absorção

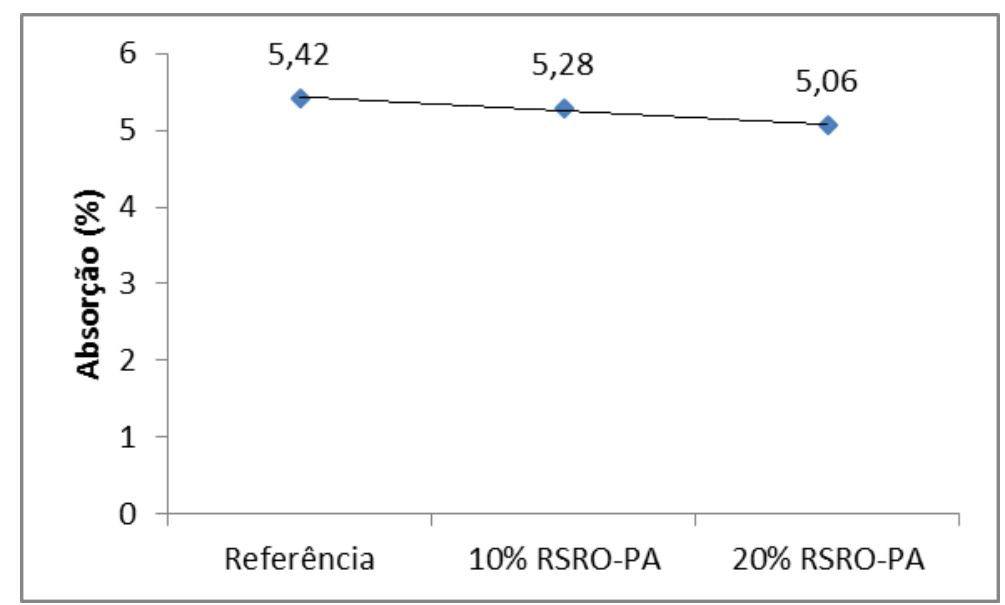

Observa-se que os concretos com RSRO-PA apresentaram menor absorção, em relação aos concretos sem resíduo. O melhor comportamento foi do concreto $20 \%$ do resíduo, que apresentou uma redução de $6,64 \%$, em relação ao concreto de referência. Este comportamento reforça a justificativa de que a utilização do RSRO proporcionou um melhor preenchimento dos espaços, densificando as misturas.

\section{CONCLUSÕES}

De acordo com os resultados pode-se concluir que a utilização de RSRO-PA em substituição à areia proporcionou melhoria nas propriedades do concreto. Houve um aumento na resistência à compressão e na resistência à tração por compressão diametral dos concretos contendo RSRO-PA. Foi verificada uma redução na absorção de água nos concretos contendo o resíduo.

Conclui-se, portanto, que a utilização de RSRO-PA como substituto parcial do agregado miúdo na produção de concretos pode ser considerada como uma alternativa para o aproveitamento do resíduo, uma vez que promoveu melhorias no comportamento mecânico do concreto além de contribuir para reduzir o impacto causado pela deposição indevida desses resíduos no ambiente e pelo grande consumo de recursos naturais na construção civil. 


\section{REFERÊNCIAS}

ASSOCIAÇÃO BRASILEIRA DE NORMAS TÉCNICAS NBR 5738. Concreto Procedimento para moldagem e cura de corpos-de-prova. Rio de Janeiro, 2003.

ASSOCIAÇÃO BRASILEIRA DE NORMAS TÉCNICAS. NBR 5739. Concreto - Ensaios de compressão de corpos-de-prova cilíndricos. Rio de Janeiro, 1994.

ASSOCIAÇÃO BRASILEIRA DE NORMAS TÉCNICAS NBR 7222. Concreto Determinação da resistência a tração por compressão diametral. Rio de Janeiro, 1994.

ASSOCIAÇÃO BRASILEIRA DE NORMAS TÉCNICAS. NBR 7251. Agregados Determinação da massa unitária e do volume de vazios. Rio de Janeiro, 1982

ASSOCIAÇÃO BRASILEIRA DE NORMAS TÉCNICAS. NBR 9778. Concreto - Argamassa e concreto endurecidos - Determinação da absorção de água, índice de vazios e massa específica. Rio de Janeiro, 2005

ASSOCIAÇÃO BRASILEIRA DE NORMAS TÉCNICAS. NBR 11578. Cimento Portland composto: Rio de Janeiro, 1991

ASSOCIAÇÃO BRASILEIRA DE NORMAS TÉCNICAS NBR 12655. Concreto de cimento Portland - Preparo, controle e recebimento - Procedimento. Rio de Janeiro, 2006.

ASSOCIAÇÃO BRASILEIRA DE NORMAS TÉCNICAS. NBR NM 52: Agregado miúdo Determinação de massa específica e massa específica aparente. Rio de Janeiro, 2002.

ASSOCIAÇÃO BRASILEIRA DE NORMAS TÉCNICAS. NBR NM 53:Agregado graúdo Determinação da massa específica, massa específica aparente e absorção de água. Rio de Janeiro, 2003.

ASSOCIAÇÃO BRASILEIRA DE NORMAS TÉCNICAS. NBR NM 67: Determinação da consistência pelo abatimento do tronco de cone. Rio de Janeiro, 1998

BARROS, P. G. S. Avaliação das propriedades de durabilidade do concreto auto-adensável obtido com resíduo de corte de mármore e granito. Maceió: Centro de Tecnologia. Universidade Federal de Alagoas, (Dissertação de Mestrado), 2008.

CHIODI FILHO, C. O setor de rochas ornamentais e de revestimento: Situação atual, demandas e perspectivas frente ao novo marco regulatório da mineração brasileira. Associação Brasileira da Indústria de Rochas Ornamentais - ABIROCHAS, São Paulo-SP, 2013.

CHIODI FILHO, C. Situação e perspectivas brasileiras no setor de rochas ornamentais e de revestimento. I Simpósio Brasileiro de Rochas Ornamentais; II Seminário de Rochas Ornamentais do Nordeste. Salvador-BA, 2001.

MANHÃES, J. P. V. T.; HOLANDA, J. N. F. Caracterização e classificação de resíduo sólido "pó de rocha granítica" gerado na indústria de rochas ornamentais. Química Nova, Vol. 31, No.6, 1301-1304, 2008.

MEHTA, P. K.; MONTEIRO, P. J. M. Concreto: Microestrutura, Propriedades e Materiais. São Paulo:Editora IBRACON,São Paulo, 2008.

MOURA, W. A.; LEITE, M. B. Estudo da viabilidade da produção de blocos com utilização de resíduo de serragem de rochas ornamentais para alvenaria de vedação.Revista Escola de Minas, v. 64, n.2, 2011.

MOURA, W. A.; LIMA, M. B. L.; GAMA, J. L. C. N. da; MORATTI, M. Resíduo de serragem de rochas ornamentais para a produção de peças pré-moldadas. Tecnologias construtivas inovadoras e gestão da cadeia produtiva. Porto Alegre : ANTAC, 2009. 
MOURA, W. A.; GONÇALVES, J. P.; LEITE, R. S. Utilização do resíduo de corte de mármore e granito em argamassas de revestimento e confecção de lajotas para piso. Feira de Santana: Sitientibus, n.26, 2002.

NEVILLE, A. M. Propriedades do concreto. São Paulo: Editora Pini, 1997.

SILVA, S. C. da. Caracterização física, química e ambiental do resíduo de granito. Potencialidades para fabricação de argamassas e de tijolos de solo-cimento. Vitória: Universidade Federal do Espírito Santo, (Dissertação de Mestrado).1998.

SILVA, R. B., SANTOS, D. O. J. Estudo de argamassas contendo resíduo de serragem de rochas ornamentais e resíduo de construção e demolição. $3^{\circ}$ Encontro Nacional Sobre Reaproveitamento de Resíduos na Construção Civil - ENARC, São Leopoldo-RS, 2013. 